availability of carbon dioxide from the soil can be increased by treatment such as stirring or introduction of potsherds, which is already known to facilitate aeration of the soil. Even more striking effects can be produced by treating the soil with small quantities of oxidising agents such as ferric oxide, potassium permanganate or hydrogen peroxide. The following observations relating to barley grown on unmanured soil are of interest.

\begin{tabular}{ccc} 
Treatment & \multicolumn{2}{c}{$\begin{array}{c}\text { Average dry weight } \\
\text { per seedling in mgm. }\end{array}$} \\
Soil alone & $\ldots$ & $38 \cdot 2$ \\
,$"+\mathrm{Fe}_{2} \mathrm{O}_{3}$ & $\ldots$ & $51 \cdot 2$ \\
,$"+\mathrm{KMnO}_{4}$ & $\ldots$ & $53 \cdot 6$ \\
,$+\mathrm{H}_{2} \mathrm{O}_{2}$ & $\ldots$ & $49 \cdot 8$
\end{tabular}

Further work is in progress, growing plants in big, specially constructed cages through which air of any desired composition and humidity can be drawn. The carbon exchanges between the atmosphere, the soil and the plant are being followed quantitatively. The practical significance of treating soil or manure with small amounts of different oxidising agents is also being investigated.

Department of Biochemistry,

V. Subrahmanyan. G. S. SiddapPa.

Indian Institute of Science, Bangalore.

1 Lundegårdh, Soil Sci., 23, 417; 1927. Biochem. Z., 194, 453; 1928. Z. Pflanz. Dünq., A. 12, 1 ; 1928. Keuhl, ibid., A. 6., 321; 1925. 1928. Z. Pflanz. Düng., A. 12, $;$; 1928. Keuhl, ibid., A. 6, 321; 1925.
Reinau, Z. angew. Chem., 39, 495; 1926. Rippel, Z. Pflanz. Düng., berg, ibid., 85. Hasse and Kirchmeyer, ibid., A, 10, 257 ; 1928.

\section{Change from Aromatic to Metallic Electrons in Organic Compounds}

THE recently published work on the crystal structures of anthracene, chrysene and dibenzanthracene $^{1}$ has shown that the graphitic structure, with all the atoms lying in one plane, and an inter. atomic distance of $1.41 \mathrm{~A}$., is already fully developed in these molecules. We may imagine graphite to be built up by successive fusion of benzene nuclei to these compounds, and the only changes in crystal structure will lie in changes of the orientation of these plane molecules to the axes of the crystal, and in changes of the distance between molecules.

The fact that electrical conductivity is shown by graphite, and not by diamond, is probably to be explained by the fact that the former consists of large plane molecules made up of fused aromatic nuclei, whereas the linkages between the carbon atoms in diamond are aliphatic in character. The work of $\mathrm{Huckel}^{2}$, and others, on the quantum mechanics of the benzene nucleus, suggests that the aromatic $p_{\mathrm{H}}$ electrons originally present in any one aromatic nucleus have a certain possibility of movement to other nuclei within the same molecule. The probability of a jump from one molecule to the next is, however, much smaller, on account of the relatively large distance between molecules.

With graphite, the distances that the electron can move within any one molecule are so large, that electrical conduction is observed when a potential is applied. Graphite is not a typical metal, however, since it has a negative temperature coefficient of resistance. This is smaller the smaller the specific resistance of the sample (for metallised graphite). If the electric conduction of graphite is due to the mobility of the
$p_{\mathrm{H}}$ electrons, as is here suggested, it will take place principally along the planes of the molecules, the transition from one plane to another being much less probable. Furthermore, since the molecules are not infinite, transitions from one molecule to the next still have to overcome an appreciable potential barrier. These facts may explain some of the anomalies of this conductor.

It is clearly important for the theory of metallic conduction to determine how large molecules made up of condensed aromatic nuclei must become, in order to show electrical conductivity. From the $\mathrm{X}$-ray evidence, the structure of molecules made up of four or five condensed nuclei is essentially the same as in graphite, and unless metallic conduction depends in a critical way upon size of the system, and number of mobile electrons, they should show a phenomenon of electron wandering, but over much smaller distances. Although the number of barriers between the molecules is too large for metallic conduction to be present, we would expect marked anomalies in certain of their physical properties. The electric polarisability would be anomalously large in directions parallel to the planes of the molecules, and marked birefringence should be evident. Similar anomalies might show themselves in the magnetic susceptibility and dielectric strength, if we compare these properties in directions parallel and perpendicular to the planes of the molecules.

The purpose of these remarks is to emphasiso that a study of the physical properties of large molecules with condensed aromatic nuclei is of considerable importance, not only in biochemistry, but also for the theory of the metallic link. Unless this depends on the presence of a critical minimum number of mobile electrons, the catalytic activity of these compounds in biological processes might possibly be ascribed to their pseudo-metallic properties.

\section{A. R. UBbezohde.}

The Clarendon Laboratory,

Oxford.

Dec. 2 .

1 J. M. Robertson, NatuRE, 132, 750, Nov. 11, 1933.

Z. Phys., 83, 632; 1933 .

\section{Light of the Night Sky and Active Nitrogen}

The afterglow in the remarkable nitrogen tube described by $\mathrm{me}^{1}$ last year has been photographed in the visible region. A reference to that paper will show that the visible spectrum of the discharge consists of the first-negative and the first-positive bands of nitrogen, with only a trace of the second-positive bands. The normal spectrum of a nitrogen discharge under the pressure and excitation conditions of my experiment consists of the second-positive and the first-positive bands. The striking change in the spectrum of the discharge itself is carried over to the afterglow.

The afterglows of nitrogen heretofore reported consist mainly of the first-positive bands which originate on the $v^{\prime}=10,11$ and 12 vibrational levels. The present afterglow is made up of bands which arise on much higher vibrational levels such as 18, 19 and 20, etc. In addition to the first-positive bands in this afterglow, there is a strong excitation of the first-negative bands. These bands are emitted by $\mathrm{N}_{2}^{+}$It may be recalled that these bands and the auroral green line compose, for the most part, the 